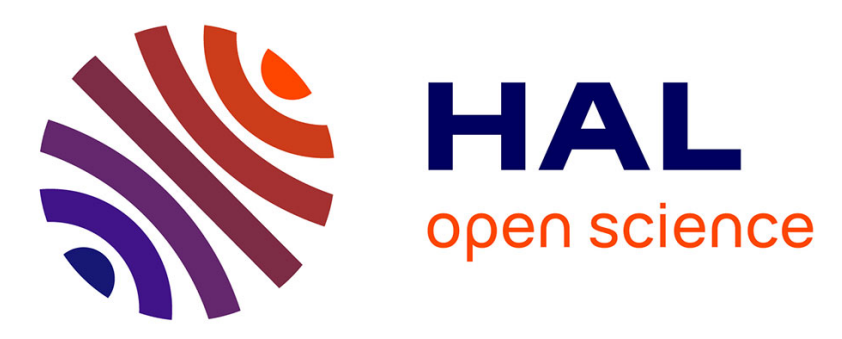

\title{
Classical Hybrid Monte-Carlo Simulation of the Interconversion of Hexabromocyclododecane Stereoisomers
}

Marcus Weber, Roland Becker, Robert Koeppen, Vedat Durmaz

\section{- To cite this version:}

Marcus Weber, Roland Becker, Robert Koeppen, Vedat Durmaz. Classical Hybrid Monte-Carlo Simulation of the Interconversion of Hexabromocyclododecane Stereoisomers. Molecular Simulation, 2008, 34 (07), pp.727-736. 10.1080/08927020802208968 . hal-00515040

\author{
HAL Id: hal-00515040 \\ https://hal.science/hal-00515040
}

Submitted on 4 Sep 2010

HAL is a multi-disciplinary open access archive for the deposit and dissemination of scientific research documents, whether they are published or not. The documents may come from teaching and research institutions in France or abroad, or from public or private research centers.
L'archive ouverte pluridisciplinaire HAL, est destinée au dépôt et à la diffusion de documents scientifiques de niveau recherche, publiés ou non, émanant des établissements d'enseignement et de recherche français ou étrangers, des laboratoires publics ou privés. 


\section{Molecular Simulation}

Journal of

Experimental Nanoscience

$\because$ Taylor \& Francis

Taglor \& Francis Group

\section{Classical Hybrid Monte-Carlo Simulation of the Interconversion of Hexabromocyclododecane Stereoisomers}

\begin{tabular}{|c|c|}
\hline Journal: & Molecular Simulation/Journal of Experimental Nanoscience \\
\hline Manuscript ID: & GMOS-2008-0067.R1 \\
\hline Journal: & Molecular Simulation \\
\hline $\begin{array}{r}\text { Date Submitted by the } \\
\text { Author: }\end{array}$ & 05-May-2008 \\
\hline Complete List of Authors: & $\begin{array}{l}\text { Weber, Marcus; Zuse-Institute Berlin (ZIB), Computational Drug } \\
\text { Design } \\
\text { Becker, Roland; Federal Institute for Materials Research and Testing } \\
\text { (BMA), Analytical Chemistry } \\
\text { Koeppen, Robert; Federal Institute for Materials Research and } \\
\text { Testing (BMA), Analytical Chemistry } \\
\text { Durmaz, Vedat; Zuse-Institute Berlin (ZIB), Computational Drug } \\
\text { Design }\end{array}$ \\
\hline Keywords: & $\mathrm{HBCD}$, isomerisation, statistical thermodynamics, kinetics \\
\hline
\end{tabular}

\section{SCHOLARONE ${ }^{m}$ Manuscripts}




\title{
Classical Hybrid Monte-Carlo Simulation of the Interconversion of Hexabromocyclododecane Stereoisomers
}

\begin{abstract}
Marcus Weber ${ }^{\mathrm{a} *}$, Roland Becker ${ }^{\mathrm{b} *}$, Vedat Durmaz ${ }^{\mathrm{a}}$ and Robert Köppen ${ }^{\mathrm{b}}$
${ }^{a}$ Department of Numerical Analysis, Computational Drug Design, Konrad-Zuse-Zentrum Berlin (ZIB), 14195 Berlin, Germany; ${ }^{\mathrm{b}}$ Department of Analytical Chemistry, Reference Materials, BAM Federal Institute for Materials Research and Testing, 12489 Berlin, Germany
\end{abstract}

The interconversion of the six main stereoisomers of the flame retardant hexabromocyclododecane (HBCD) is investigated by means of statistical thermodynamics using classical force-fields. $( \pm)-\alpha-,( \pm)-\beta-$, and $( \pm)-\gamma-$ HBCD interconvert by swapping of absolute configurations on the three different (BrHC-CHBr)-moieties. The approach avoids saddle-point energy computations but relies on classical thermodynamic simulation and pursues three consecutive steps. First, the application of classical hybrid Monte-Carlo simulations for quantum mechanical processes is justified. Second, the problem of insufficient convergence properties of hybrid Monte-Carlo methods for the generation of low temperature canonical ensembles is solved by an interpolation approach. Third, it is shown how free energy differences among stereoisomers are derived and how they can be used for the computation of interconversion rates. The simulation results confirm the experimentally observed interconversion rates and correctly identify $\alpha$-HBCD as a thermodynamical sink in the oscillating mixture of stereoisomers.

Keywords: HBCD; isomerisation; statistical thermodynamics; kinetics

*Corresponding authors. Email: weber@zib.de, roland.becker@bam.de

\section{Introduction}

Molecular dynamics simulations based on classical force fields can be used to determine the rate of conformational changes inside the conformational space of small molecules. However, the classical description of molecular motion fails if the substance undergoes intramolecular isomerisation. This is a 
quantum mechanical process and the timescale of such an interconversion is often far away from the step length of quantum mechanical simulations. In this paper, we present an interesting example for such a situation with practical implications, namely, 1,2,5,6,9,10-hexabromocyclododecane (HBCD), as shown in Figure 1. Our goal was to find a classical approach for the qualitative estimation of the interconversion rates, making simulation as simple as possible and, therefore, reproducible. Since single-point quantum chemical calculations often yield poor prediction results for relevant physical properties of substances with considerable conformational flexibility, thermodynamical methods for the investigation of the behavior of HBCD were adopted. Thus, mainly three ideas are presented in this paper:

1. It is shown how to investigate the interconversion processes between the HBCD isomers using classical simulation methods.

2. Section Reweighting Formula provides a method to solve the trapping problem of low-temperature molecular simulations by a reweighting approach.

3. Interconversion rates can be computed, using free energy differences and a steady-state approximation, as described in section Rate Matrix Computation.

As a consequence of the employed approach, the interconversion rates are not estimated using saddle-point energy computations but they are derived from classical thermodynamics simulations. The calculations are based on an interpolation of a converging high-temperature simulation of the (+)-enantiomers of each of the three diastereomers. The presented approach might trigger further developments in this field.

\section{The isomerism of $\mathrm{HBCD}$ and the interconversion mechanism}

HBCD is one of the major flame retardant additives to plastics and it is increasingly found in trace amounts in the environment, biota, and humans [1-4]. Therefore, HBCD is currently one of the emerging environmental analytes of interest. There is evidence for activity of HBCD as endocrine disruptor [5] and an EU risk assessment is under way. Technical HBCD consists mainly of three diastereomeric pairs of enantiomers (Figure 1). 
<smiles>Br[C@H]1CC[C@H](Br)[C@@H](Br)CC[C@H](Br)[C@@H](Br)CC[C@@H]1Br</smiles><smiles>Br[C@H]1CC[C@H](Br)[C@@H](Br)CC[C@H](Br)[C@@H](Br)CC[C@@H]1Br</smiles><smiles>Br[C@H]1CC[C@H](Br)[C@@H](Br)CC[C@H](Br)[C@@H](Br)CC[C@@H]1Br</smiles><smiles>Br[C@H]1CC[C@H](Br)[C@@H](Br)CC[C@H](Br)[C@@H](Br)CC[C@@H]1Br</smiles><smiles>Br[C@H]1CC[C@H](Br)[C@@H](Br)CC[C@H](Br)[C@@H](Br)CC[C@@H]1Br</smiles><smiles>Br[C@H]1CC[C@H](Br)[C@@H](Br)CC[C@H](Br)[C@@H](Br)CC[C@@H]1Br</smiles>

Figure 1. Different stereoisomers of technical HBCD: Top row from left to right: $(+)-\alpha$-HBCD, $(+)-\beta$-HBCD, $(+)-\gamma$-HBCD. Bottom row from left to right: (-)- $\alpha$-HBCD, (-)- $\beta$-HBCD, $(-)-\gamma$-HBCD

The absolute configurations of the enantiomers were only recently correlated with their order of chromatographic elution [6]. We are interested in the interconversion of the HBCD stereoisomers shown in Figure 1, because it is important to understand their behaviour in technical processes and gas chromatographic analysis. Furthermore, the diastereomeric and enantiomeric pattern in biological samples differ from that of the technical mixture and a biologically induced interconversion of HBCD at ambient temperature was suggested $[4,7]$.

The thermally induced interconversion of HBCD stereoisomers was first reported by Peled [8], confirmed later [9], and recently studied in detail $[10,11]$. It was shown experimentally that any given mixture of $\alpha$ $\mathrm{HBCD}, \beta$-HBCD, and $\gamma$-HBCD moves towards an equilibrium dominated by $\alpha$-HBCD $[8,9,10]$. Experiments with neat (+)- $\alpha$-HBCD, (+)- $\beta$-HBCD, or $(+)-\gamma$-HBCD revealed that the reaction mixtures contain exclusively the six stereoisomers shown in Figure 1 [10] and that their isomerizations proceed as depicted in Figure 2. 


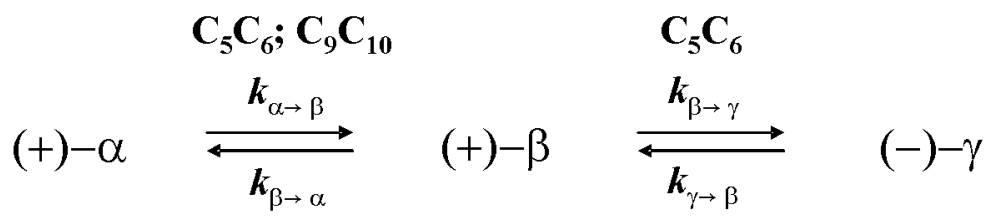

$$
\begin{aligned}
& \begin{array}{l}
\boldsymbol{k}_{\alpha \rightarrow \gamma} \\
\mathbf{C}_{1} \mathbf{C}_{2}
\end{array}\left|\begin{array}{rr}
\boldsymbol{k}_{\gamma \rightarrow \alpha} & \mathbf{C}_{1} \mathbf{C}_{2}
\end{array}\right| \begin{array}{lll}
\boldsymbol{k}_{\beta \rightarrow \beta} & & \boldsymbol{k}_{\gamma \rightarrow \alpha} \\
& \mathbf{C}_{5} \mathbf{C}_{6} ; \mathbf{C}_{9} \mathbf{C}_{10}
\end{array} \| \boldsymbol{k}_{\alpha \rightarrow \gamma} \\
& (+)-\gamma \underset{\boldsymbol{k}_{\beta \rightarrow \gamma}}{\stackrel{\boldsymbol{k}_{\gamma \rightarrow \beta}}{\rightleftarrows}}(-)-\beta \quad \underset{\boldsymbol{k}_{\alpha \rightarrow \beta}}{\stackrel{\boldsymbol{k}_{\beta \rightarrow \alpha}}{\rightleftarrows}}(-)-\alpha
\end{aligned}
$$

Figure 2. Experimentally observed interconversion reactions of the HBCD isomers connected to the three respectively involved (BrHC-CHBr)-moieties $\left(\mathrm{C}_{1} \mathrm{C}_{2}, \mathrm{C}_{5} \mathrm{C}_{6}\right.$, and $\left.\mathrm{C}_{9} \mathrm{C}_{10}\right)$.

The mechanism of the transition between these structures is shown in Figure 3. The absolute configurations of two neighbouring stereocenters are simultaneously inverted via a cyclic-concerted transition state which was so far only investigated on variously substituted 1,2-dibromocyclohexanes [12-14] and 1,2dibromocyclooctanes [15].

\section{$\mathrm{C}_{1} \mathrm{C}_{2}$ :}

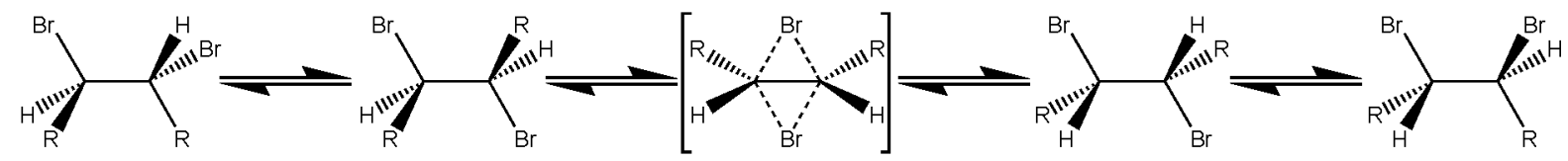

$(\mathrm{R}, \mathrm{R})$ gauche

(R,R) anti<smiles>BrC1C2CC3C1CC3C2Br</smiles><smiles></smiles>

$(\mathrm{S}, \mathrm{S})$ anti

(S,S) gauche<smiles>[R]C1C(Br)C2CC3C(Br)C(C2Br)C13</smiles><smiles></smiles>

A

$\mathbf{A B}^{\ddagger}$

\section{$\mathrm{C}_{5} \mathrm{C}_{6} ; \mathrm{C}_{9} \mathrm{C}_{10}$ :}

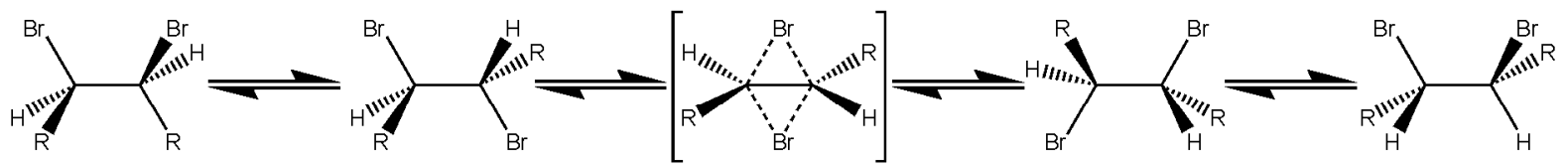

(R,S) gauche<smiles>[R]C1C2CC3C(Br)C2CC(Br)(C3[R])C1Br</smiles>

$(\mathrm{S}, \mathrm{R}) a n t i$

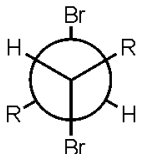

$\mathrm{CD}^{\ddagger}$
(S,R) gauche<smiles>[R]C1C(Br)C2C(Br)C3CC1C32</smiles>

C

Figure 3. Stereochemistry of the interconversion of $H B C D$. The $(R, R) \leftrightarrow(S, S)$ and $(R, S) \leftrightarrow(S, R)$ isomerisations require different orientation of the ring carbons $\mathrm{R}$ in the crucial anti conformations and transition states $\mathrm{AB}$ and $\mathrm{CD}$. 
Any interconversion requires anti-position of the two vicinal bromine atoms involved. This prerequisite leads to different consequences for the three (BrHC-CHBr)-moieties depending on their relative configuration. In case of $\mathrm{C}_{1} \mathrm{C}_{2}$ the ring carbons $\mathrm{R}$ are forced in gauche-position while in case of $\mathrm{C}_{5} \mathrm{C}_{6}$ and $\mathrm{C}_{9} \mathrm{C}_{10}$ they have to adopt anti-position. Though the anti alignment might appear energetically more favourable at first sight it should be noted that the $\mathrm{R}$ are part of the relative small cyclododecane ring and therefore the dihedral angle of $180^{\circ}$ in $\mathrm{C}, \mathrm{D}$, and $\mathrm{CD}$ is likely to cause considerable strain which is avoided in $\mathrm{A}, \mathrm{B}$, and $\mathrm{AB}$. This structural similarity between the anti conformations and the respective transition states $\mathrm{AB}$ and $\mathrm{CD}$ leads to the idea that classical simulation of the free energy differences of the respective gauche and anti conformational spaces can be used to characterise the interconversion processes qualitatively. For the definition of anti and gauche conformational states see Figure 4.

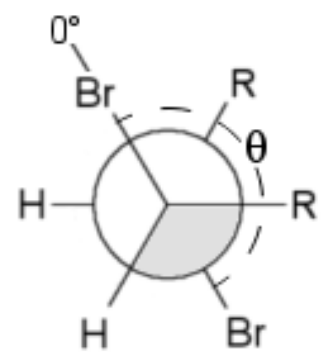

\section{$|\theta|>120$ : anti position \\ $|\theta|<120$ : gauche position}

Figure 4. Definition of anti and gauche conformational states.

These considerations imply that the additional barrier between any given anti conformation and the respective transition state is not decisive. It is rather assumed that the lower the barrier between the gauche and anti conformations of two given vicinal bromines $\left(\mathrm{C}_{1} \mathrm{C}_{2}, \mathrm{C}_{5} \mathrm{C}_{6}\right.$, or $\left.\mathrm{C}_{9} \mathrm{C}_{10}\right)$ the faster the respective conversion will be.

Since the transition states $\mathrm{AB}$ and $\mathrm{CD}^{\prime}$ cannot trivially be treated by means of classical force fields their consideration would make a simulation technically unfeasible.

In the following, the respective parts of the configurational space corresponding to the gauche- and the antipositions are compared via computer simulation for each (BrHC-CHBr)-moiety of every diastereomer and expressed in terms of free energy differences. The simulations are based on the Boltzmann distribution (canonical ensemble) of states which is the most likely distribution of states at constant temperature, constant number of particles, and constant volume. In order to compare simulations with experiments carried out with (+) $-\gamma$-HBCD [10] and since the interconversion only takes place in the liquid phase, the melting point of $(+)-\gamma-$ $\mathrm{HBCD}$ at $433 \mathrm{~K}$ is the temperature of interest. However, the simulations were performed for the vacuum as a simplified approximation and the mutual interactions of the HBCD molecules in the liquid state were neglected. It should be noted that the intention was to explore the prospects of the adopted approach with regard to a qualitative correlation of simulated with observed properties of the oscillating mixture of stereoisomers namely the relative ratio of the interconversion rates $k$ and the predominance of certain stereoisomers at equilibrium. We know that the HBCD stereoisomers have different polarities, volumes, shapes, and may thus be differently solvated (the solvent is HBCD itself). The vacuum approximation is a first approach in order to simulate reaction rates in Figure 2 qualitatively. Even if we could model HBCD as a liquid, a sufficient and equilibrated simulation of this complex model can not be expected within reasonable time with reasonable computational costs. 


\section{Experimental results of the interconversion}

The experimental set-up for the kinetic analysis of the isomerisation of HBCD is discussed in detail elsewhere [10]. In brief, pure (+)- $\gamma$-HBCD was exposed to $433 \mathrm{~K}$ for different periods between two and 60 minutes and the concentrations of all six stereoisomers (Figure 1) were determined for each point in time by liquid chromatography with diode array detection and using a chiral column. Since no other compound including different HBCD isomers had been detected, the summed concentration of the six HBCD stereoisomers measured at a particular time was normalized to $100 \%$ for each point in time. Then, the molar fraction of each diastereomer was calculated as molar percent (mol-\%). The Mathematica 4.0 software package from Wolfram Research, Inc. (USA) was used for a numerical estimation of the rate constants k. For this purpose, the time dependent concentration vector $x \in I R^{6}$ was modeled as a solution of a rate equation.

$\frac{d x(t)}{d t}=Q^{T} x(t)$

where $Q \in I R^{6 \times 6}$ is the corresponding rate matrix, and $x$ is the vector of the concentrations of the six species of Figure 1 (in the corresponding order: $(+)-\alpha,(+)-\beta,(+)-\gamma,(-)-\alpha,(-)-\beta,(-)-\gamma)$. The least squares fit of the experimental data corresponds to the following experimental (exp) rate matrix result:

$$
Q^{\exp }=0.001 \cdot\left(\begin{array}{cccccc}
-0.161 & 0.019 & 0.142 & 0 & 0 & 0 \\
0.12 & -1.39 & 0 & 0 & 1.1 & 0.17 \\
1.5 & 0 & -1.646 & 0 & 1.460 & 0 \\
0 & 0 & 0 & -0.161 & 0.019 & 0.142 \\
0 & 1.1 & 0.17 & 0.12 & -1.39 & 0 \\
0 & 0.146 & 0 & 1.5 & 0 & -1.64
\end{array}\right)
$$

These values correspond to the experimental interconversion rates $\left(\right.$ mol- $\% \mathrm{~s}^{-1}$ ) $\mathrm{k}_{\alpha \rightarrow \gamma}=1.42 \cdot 10^{-4}, \mathrm{k}_{\alpha \rightarrow \beta}=$ $1.88 \cdot 10^{-5}, \mathrm{k}_{\beta \rightarrow \alpha}=1.2 \cdot 10^{-4}, \mathrm{k}_{\beta \rightarrow \gamma}=1.7 \cdot 10^{-4}, \mathrm{k}_{\gamma \rightarrow \beta}=1.46 \cdot 10^{-4}, \mathrm{k}_{\gamma \rightarrow \alpha}=1.5 \cdot 10^{-3}, \mathrm{k}_{\beta \rightarrow \beta}=1.1 \cdot 10^{-3}$ in [10]. The corresponding time-dependent concentration plots are shown in Figure 5. It should be noted that the isomerization of $\gamma$ to $\alpha$ and the racemization of $\beta$ are the fastest reactions in accordance with the conjecture that the activation energy for the anti positions $A$ and $B$ in case of the isomerisation $(R, R) \leftrightarrow(S, S)$ on $C_{1} C_{2}$ is lower than that of the $(\mathrm{R}, \mathrm{S}) \leftrightarrow(\mathrm{S}, \mathrm{R})$ isomerization occurring on $\mathrm{C}_{5} \mathrm{C}_{6}$ and $\mathrm{C}_{9} \mathrm{C}_{10}$ (Figure 3). 

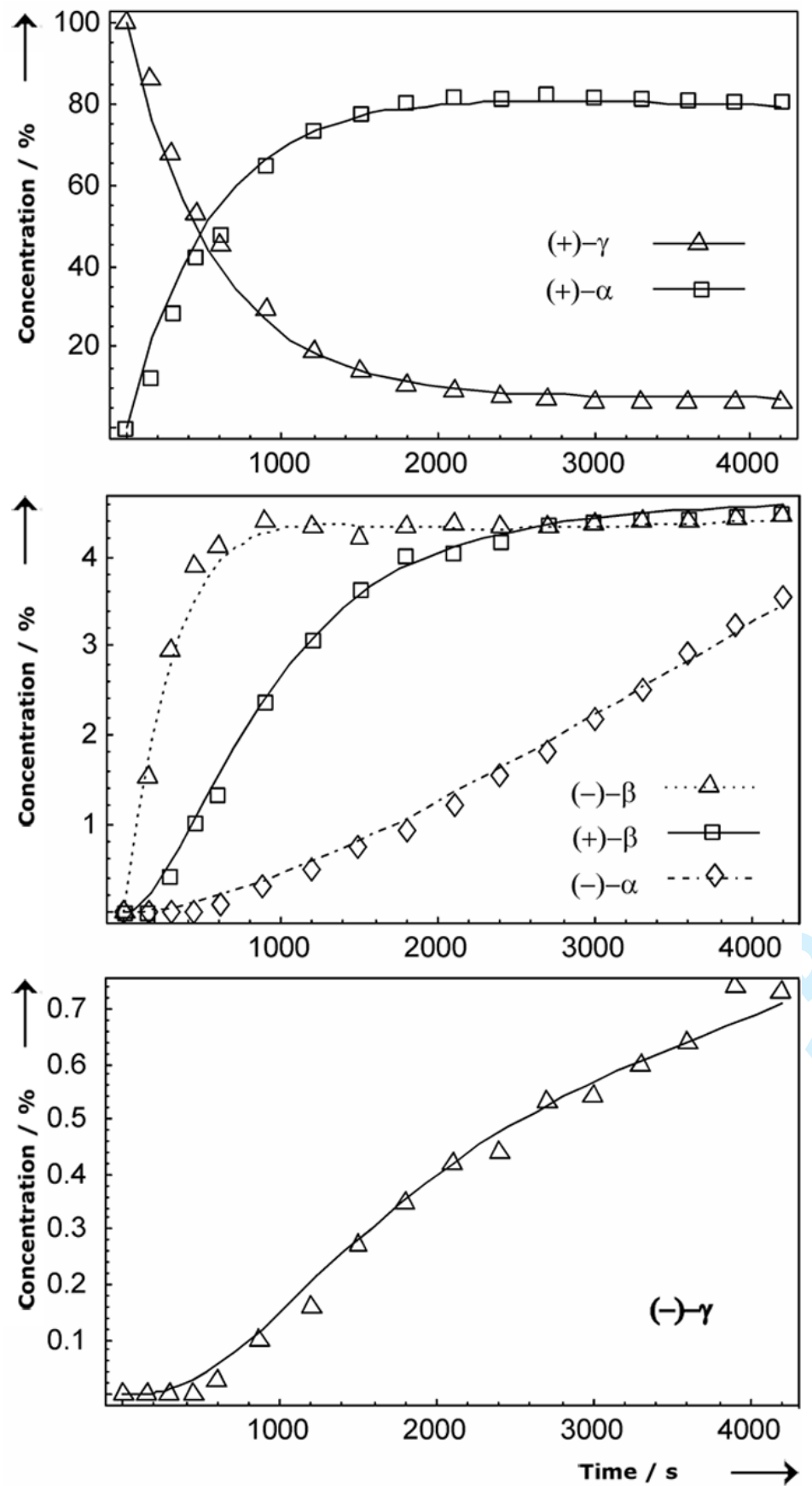

Figure 5. Interconversion experiment starting with $100 \%(+)-\gamma$-HBCD.

\section{Hybrid Monte-Carlo simulation}

In the Boltzmann distribution of states at temperature $T$ the probability $\pi(q)$ for a conformation $q \in \Omega$ of the conformational space $\Omega$ is proportional to

$\pi(q) \propto \exp \left(-\beta V_{p o t}(q)\right)$ 
with the inverse temperature

$\beta=\left(T \cdot 0.008314403 \frac{\mathrm{kJ}}{\mathrm{mol} \cdot \mathrm{K}}\right)^{-1} \Rightarrow \beta \propto T^{-1}$

In (2), $V_{\text {pot }}: \Omega \rightarrow I R$ is the potential energy of the conformational states. For our classical simulations, this function is given by the Merck Molecular Force Field which has been designed for small molecules like HBCD [16, 17]. Note, that the transition in Figure 2 is not modelled by the classical simulation. Via simulation, we estimate the part of the state space of each of the stereoisomers which can be seen as activated (anti-positioned bromine atoms, see Figure 4). Only if the molecule is in one of the activated states, it can interconvert into another stereoisomer. The parameters of the Merck Molecular Force Field have been fitted to quantum chemical calculations, especially according to rotational barriers of dihedral angles. Thus, we have decided to use this special potential energy function. In order to generate a set of states (a trajectory of states) distributed according to (2), we applied the hybrid Monte-Carlo method (HMC) [18, 19]. Analogously to Metropolis Monte-Carlo, the HMC algorithm consists of a proposal step and an acceptance step. The proposal step is a short time (78 fs, using a velocity verlet integrator [20] with a time-step of $1.3 \mathrm{fs}$ ) molecular dynamics (MD) simulation. The initial momenta for the MD part of the algorithm are taken from the Boltzmann distribution of momenta at the given temperature. By using this special kind of Monte-Carlo method, the cyclic structure of HBCD is preserved during the proposal step, there is no need for a special treatment of cyclic molecules. Unfortunately, the HMC-method rarely overcomes high potential energy barriers [21, 22]. Only if the kinetic energy of the initial momenta is high enough, the HMC-method is able to reach the important parts of the conformational space.

For this reason, we simulated the three molecules at an artificially high temperature $T=1500 \mathrm{~K}$ with $\beta_{0}=$ $0.08018 \mathrm{~mol} \mathrm{~kJ}^{-1}$. The convergence was tested according to Gelman and Rubin [23, 24]. It was seen that 10,000 steps were not sufficient, but convergence was reached after five times 100,000 steps for each of the (+)-structures. Since we are interested in the thermodynamics of the molecules at $T=433 \mathrm{~K}$ with $\beta_{1}=$ $0.27777 \mathrm{~mol} \mathrm{~kJ}^{-1}$, we derived, in a first step, thermodynamical values from the HMC sampling for $T=1500$ $\mathrm{K}$. Afterwards, these quantities were reweighted. One thermodynamic value is the free energy $A$ of the system. It is given by the partition function

$$
A(\beta)=-\frac{1}{\beta} \ln \left(\int_{\Omega} \exp \left(-\beta V_{p o t}(q)\right) d q\right) .
$$

In equation (4), the kinetic energy part of $A$ is missing. This simplification is possible, because we are only interested in free energy differences. Furthermore, potential and kinetic energy are separable and the kinetic energy part is identical for the observed subsystems. Though, the integral in (4) can not be approximated by numerical methods, ratios of integrals over certain subsets of $\Omega$ can be approximated, i.e. free energy differences can be computed. For a given (BrHC-CHBr)-moiety, we are interested in the portion of states that can be seen as anti compared to the portion of states that are gauche, with respect to the corresponding dihedral angle $\theta$. In the present application, dihedral angles $120^{\circ} \geq|\theta| \geq-120^{\circ}$ are defined as gauche and dihedral angles $|\theta|>120^{\circ}$ are defined as anti.

With this separation of states, we determined free energy differences from the HMC-sampling by counting the different states $N_{\text {anti }}$ and $N_{\text {gauche }}$, respectively, and applying

$$
\Delta_{g a} A(\beta) \approx-\frac{1}{\beta} \ln \left(\frac{N_{\text {bauche }}}{N_{\text {anti }}}\right) .
$$


The results are given in Table 1 . The more negative the free energy difference $\Delta_{\mathrm{ga}} A$ between anti- (a) and gauche-conformations $(\mathrm{g})$ the more the latter are preferred.

\begin{tabular}{|c|c|c|c|c|c|c|}
\hline \multicolumn{7}{|c|}{$\begin{array}{l}\text { Table 1. Free energy differences } \Delta_{\mathrm{ga}} A\left(\mathrm{~kJ} \mathrm{~mol}^{-1}\right) \text { and mean } \\
\text { potential energy differences } \Delta_{\mathrm{ga}}<V_{\mathrm{pot}}>\left(\mathrm{kJ} \mathrm{mol}^{-1}\right) \text { between } \\
\text { gauche- and anti-position for the three }(\mathrm{BrHC}-\mathrm{CHBr})- \\
\text { moieties as well as the mean potential energy }<V_{\mathrm{pot}}>(\mathrm{kJ} \text { mol } \\
\left.{ }^{1}\right) \text { of each (+)-diastereomer of } \mathrm{HBCD} \text { at } T=1500 \mathrm{~K} \text {. }\end{array}$} \\
\hline & \multicolumn{2}{|c|}{$(+)-\alpha$-HBCD } & \multicolumn{2}{|c|}{$(+)-\beta$-HBCD } & \multicolumn{2}{|c|}{$(+)-\gamma$-HBCD } \\
\hline \multirow[t]{2}{*}{$<V_{\text {pot }}>$} & \multicolumn{2}{|c|}{921} & \multicolumn{2}{|c|}{931} & \multicolumn{2}{|c|}{926} \\
\hline & $\Delta_{\mathrm{ga}} A$ & $\begin{array}{c}\Delta_{\mathrm{ga}}<V_{\mathrm{p}} \\
{ }_{\mathrm{ot}}>\end{array}$ & $\Delta_{\mathrm{ga}} A$ & $\begin{array}{c}\Delta_{\mathrm{ga}}<V_{\mathrm{po}} \\
\mathrm{p}\end{array}$ & & $\begin{array}{c}\Delta_{\mathrm{ga}}<V_{\text {pot }} \\
>\end{array}$ \\
\hline $\mathrm{C}_{1} \mathrm{C}_{2}$ & -17 & -8 & -14 & 0 & -10 & 5 \\
\hline $\mathrm{C}_{5} \mathrm{C}_{6}$ & -40 & -26 & -36 & -20 & -41 & -28 \\
\hline $\mathrm{C}_{9} \mathrm{C}_{10}$ & -40 & -25 & & -17 & -41 & -27 \\
\hline
\end{tabular}

Another useful value can be derived from the simulations: The mean potential energy value

$$
\left\langle V_{p o t}\right\rangle(\beta)=\int_{\Omega} V_{p o t}(q) \frac{\exp \left(-\beta V_{p o t}(q)\right)}{\int_{\Omega} \exp \left(-\beta V_{p o t}(q)\right) d q} d q
$$

The inner energy $<V_{\text {tot }}>$ of the system is the sum

$$
\left\langle V_{\text {tot }}\right\rangle=\left\langle V_{p o t}\right\rangle+\left\langle V_{\text {kin }}\right\rangle
$$

of the mean potential and the mean kinetic energy. Numerically, $\left\langle V_{\text {pot }}\right\rangle$ can be approximated by the mean of the potential energy values of the HMC-sampling, and analogously the mean potential energy differences can be computed from the two subsets comprising all anti- and gauche-conformations, respectively (Table 1).

\section{Reweighting formula}

The high-temperature sampling with $T=1500 \mathrm{~K}$ needs to be reweighted to $T=433 \mathrm{~K}$. The term "reweighting" is mostly used for the following method: A statistical weight is assigned to every generated data-point from the high-temperature sampling. One tries to adjust these weights in order to approximate the low-temperature distribution of energies.

This procedure often fails: In high temperature samplings the potential energy values are distributed in a broader range with a higher mean value compared to a low-temperature sampling, see Figure 6. 
Most of the sampling points have a high potential energy value. If we aim at a reweighting of the sampling points in order to get the low-temperature distribution, the low-energy points of the high-temperature sampling are weighted up statistically. Therefore, point-wise reweighting schemes only make use of the sampling points located in the small overlap region of the high-temperature and the low-temperature distribution. The occupation of this region depends on the input parameters of the algorithms very sensitively. To the common opinion, reweighting is only possible for sufficiently overlapping distributions. This opinion led to many sophisticated sampling methods in the past. The most prominent one is replica exchange [25], and other examples can be found in modern textbooks [26]. Here, it is not important to approximate the complete distribution of states at the lower temperature, but it is sufficient to know, how the mean value in Figure 6 depends on the temperature. Instead of a point-wise reweighting scheme we present a thermodynamical approach. For the purpose of simplicity the mean potential energy $\left\langle V_{\text {pot }}\right\rangle$ is abbreviated with $U$ in the followings, and as an approximation, $U$ is assumed to depend linearly on the temperature $T$ of the considered system. Therefore, $\Delta U(\beta)$ goes with $\beta^{-1}$, due to equation (3) which leads us to

$\Delta U(\beta)=\frac{\left(\Delta U\left(\beta_{0}\right)-\Delta U(\infty)\right) \beta_{0}}{\beta}+\Delta U(\infty)$

Since the mean kinetic energy $\left\langle V_{\text {kin }}>\right.$ in (7) depends linearly on the temperature, the assumption (8) is equivalent to a linear model for the inner energy $\left\langle V_{\text {tot }}\right\rangle$ of the system. Furthermore, assuming a linear model for the inner energy in reality would mean that the heat capacity is a temperature-independent constant. This assumption is not true, but mostly according to quantum effects which are not part of the classical model used here. From the classical viewpoint, (8) is exact, if the potential energy $V_{\text {pot }}$ is a quadratic function in $\Omega$. Formula (8) is a good approximation if the conformational space $\Omega$ can be decomposed, such that each part of this decomposition has a Gaussian-like Boltzmann distribution. This is a widely used assumption in many applications. More details can be found in the Appendix. In order to apply (8), we have to compute the limit $\Delta U(\infty)$. With increasing $\beta$, the Boltzmann distribution more and more focuses on the global optimum of the system. Since the conformational space was split into two subsystems of anti-and gauche-states, the limit of $\Delta U$ for increasing $\beta$ is equivalent to the difference $\Delta_{\mathrm{ga}} V_{\mathrm{pot}}$ of the lowest potential energy values of the two subsystems, i.e. $\Delta_{\mathrm{ga}} V_{\text {pot }}{ }^{0}=\Delta_{\mathrm{ga}} U(\infty)$. The entries in Table 2 are the results of local minimisations (using the conjugate gradient method [27]) applied to each point of the trajectories of the given high-temperature HMCsamplings. The gauche-position of the bromine atoms is always preferred. 
Table 2. Lowest potential energy values $\mathrm{V}_{\text {pot }}{ }^{0}$ for the anti- and gauche- conformations and their differences $\Delta_{\mathrm{ga}}<V_{\mathrm{pot}}{ }^{0}>\left(\mathrm{kJ} \mathrm{mol}^{-1}\right)$ for the three (BrHC-CHBr)-moieties of each (+)diastereomer of $\mathrm{HBCD}$.

\begin{tabular}{|c|ccc|ccc|ccc|} 
& \multicolumn{3}{|c|}{$(+)-\alpha$-HBCD } & \multicolumn{3}{c|}{$(+)-\beta$-HBCD } & \multicolumn{3}{c|}{$(+)-\gamma-\mathrm{HBCD}$} \\
\hline & gauche & anti & $\Delta_{\mathrm{ga}}\left\langle V_{\text {pot }}{ }^{0}>\right.$ & gauche & anti & $\Delta_{\text {ga }}<V_{\text {pot }}{ }^{0}>$ & gauche & anti & $\Delta_{\text {ga }}<V_{\text {pot }}{ }^{0}>$ \\
\hline $\mathrm{C}_{1} \mathrm{C}_{2}$ & 239 & 253 & -14 & 249 & 264 & -15 & 257 & 257 & 0 \\
$\mathrm{C}_{5} \mathrm{C}_{6}$ & 239 & 272 & -33 & 249 & 285 & -36 & 257 & 275 & -18 \\
$\mathrm{C}_{9} \mathrm{C}_{10}$ & 239 & 272 & -33 & 249 & 276 & -27 & 257 & 275 & -18 \\
\hline
\end{tabular}

This corresponds to the experiment: All known crystal structures of HBCD stereoisomers display all-gauche configuration of all vicinal bromine atoms [6, 28-30]. The mean potential energy values $\left\langle V_{\text {pot }}\right\rangle$ according to the linear model (8) are presented in Table 3.

\begin{tabular}{|c|c|c|c|c|c|c|}
\hline \multicolumn{7}{|c|}{$\begin{array}{l}\text { Table 3. Free energy differences } \Delta_{\mathrm{ga}} A\left(\mathrm{~kJ} \mathrm{~mol}^{-1}\right) \text { and mean potential energy }\left\langle V_{\mathrm{pot}}>\left(\mathrm{kJ} \mathrm{mol}^{-1}\right) \text { of }\right. \\
\text { HBCD at } T=433 \mathrm{~K} \text {. }\end{array}$} \\
\hline & \multicolumn{2}{|c|}{$(+)-\alpha-\mathrm{HBCD}$} & \multicolumn{2}{|c|}{$(+)-\beta$-HBCD } & \multicolumn{2}{|c|}{$(+)-\gamma$-HBCD } \\
\hline \multirow{2}{*}{$<V_{\text {pot }}>$} & & 436 & 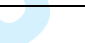 & 446 & & 450 \\
\hline & $\Delta_{\mathrm{ga}} A$ & Interconverts to: & $\Delta_{\mathrm{ga}} A$ & $\begin{array}{l}\text { Interconverts } \\
\quad \text { to: }\end{array}$ & $\Delta_{\mathrm{ga}} A$ & Interconverts to: \\
\hline $\mathrm{C}_{1} \mathrm{C}_{2}$ & -13 & $(+)-\gamma$ & & $(+)-\beta$ & -1 & $(+)-\alpha$ \\
\hline $\mathrm{C}_{5} \mathrm{C}_{6}$ & -33 & $(+)-\beta$ & -30 & $(+)-\gamma$ & -28 & $(+)-\beta$ \\
\hline $\mathrm{C}_{9} \mathrm{C}_{10}$ & -32 & $(+)-\beta$ & -25 & $(+)-\alpha$ & -28 & $(+)-\beta$ \\
\hline
\end{tabular}

In order to determine interconversion rates, we are not interested in the mean potential energy values but in free energy differences $\Delta_{\mathrm{ga}} A(\beta)$. The $\beta$-dependency of $A$ can be calculated by differentiating (4) and inserting (6):

$\frac{d}{d \beta} \Delta_{g a} A(\beta)=-\frac{1}{\beta} \Delta_{g a} A(\beta)+\frac{1}{\beta} \Delta_{g a}\left\langle V_{p o t}\right\rangle(\beta)$.

Using the linear model (8) for $U$ provides

$\frac{d}{d \beta} \Delta_{g a} A(\beta)=-\frac{1}{\beta} \Delta_{g a} A(\beta)+\frac{\left(\Delta U\left(\beta_{0}\right)-\Delta U(\infty)\right) \beta_{0}}{\beta^{2}}+\frac{\Delta U(\infty)}{\beta}$.

The ordinary differential equation (10) can be solved analytically, such that

$\Delta_{g a} A(\beta)=\frac{1}{\beta}\left(\left(\beta-\beta_{0}\right) \Delta U(\infty)+\ln \left(\frac{\beta}{\beta_{0}}\right) \beta_{0}\left(\Delta U\left(\beta_{0}\right)-\Delta U(\infty)\right)+\beta_{0} \Delta_{g a} A\left(\beta_{0}\right)\right)$

for an initial value $\Delta_{\mathrm{ga}} A\left(\beta_{0}\right)$ which can be found in Table 1 .

Via formula (11) and the corresponding values for $\Delta U\left(\beta_{0}\right), \Delta U(\infty)$, and $\Delta_{\mathrm{ga}} A\left(\beta_{0}\right)$ the free energy differences can be computed for $T=433 \mathrm{~K}$. The results are given in Table 3 . In contrast to a point-wise reweighting scheme, formula (11) is robust against small perturbations of the input data. 


\section{Rate matrix computation}

The results of the reweighting process are shown in Table 3. They lead to the following questions: How do these simulation results fit to the experimental results? Do they reflect the qualitative behavior of the HBCDsystem? In order to answer these questions, the meaning of the simulation results for the qualitative behavior of the HBCD-system is visualized in the followings. From a mathematical point of view, we will apply the Arrhenius equation [31]:

$k \propto \exp \left(-\beta \Delta_{a g} A\right)$

In this approach, the interconversion rate $k$ is proportional to the Boltzmann expression of the activation energy. With $\Delta_{\mathrm{ag}} A=-\Delta_{\mathrm{ga}} A$, the free energy differences in Table 3 allow to estimate these activation energies. On the basis of these considerations, a rate matrix $Q \in I R^{6 \times 6}$ is constructed for the six stereoisomers (in the corresponding order: (+)- $\alpha,(+)-\beta,(+)-\gamma,(-)-\alpha,(-)-\beta,(-)-\gamma)$. The model is based on the rate equation (1) with the concentration vector $x \in I R^{6}$. It is known from theory [32, 33, 34] that the rate matrix $Q$ of this Markov process can be written in the form

$Q=R(K-i d)$

where $i d$ is the six-dimensional unit matrix, $K \in I R^{6 \times 6}$ is the embedded Markov chain and $R \in I R^{6 \times 6}$ is a diagonal matrix of rate factors. On the basis of the free energy differences and (12), the embedded Markov chain can be computed by inserting the values of the Arrhenius equation into a matrix and rescaling the rows, such that the row sums equal 1 . For HBCD, it has to be taken into account that the interconversion of $\alpha$ - and $\gamma$-HBCD into $\beta$-HBCD occurs identically on $\mathrm{C}_{5} \mathrm{C}_{6}$ and $\mathrm{C}_{9} \mathrm{C}_{10}$. In consequence, the respective Arrhenius expression $k$ has to be multiplied with factor 2 :

$$
K=\left(\begin{array}{cccccc}
0 & 0.008 & 0.992 & 0 & 0 & 0 \\
0.013 & 0 & 0 & 0 & 0.986 & 0.003 \\
0.999 & 0 & 0 & 0 & 0.001 & 0 \\
0 & 0 & 0 & 0 & 0.008 & 0.992 \\
0 & 0.986 & 0.003 & 0.011 & 0 & 0 \\
0 & 0.001 & 0 & 0.999 & 0 & 0
\end{array}\right) .
$$

The rate factors can be computed, considering the equilibrium concentrations of the six species as a steadystate of equation (1). The determination of the steady-state vector $\pi \in I R^{6}$ doesn't require experimental data. The equilibrium concentrations can be estimated by the mean potential energy values in Table 3 and the Boltzmann expression (2), instead (replacing $V_{\text {pot }}$ by $\left\langle V_{\text {pot }}\right\rangle$ ). This results in the steady-sate vector

$\pi^{T}=\left(\begin{array}{llllll}0.462 & 0.029 & 0.009 & 0.462 & 0.029 & 0.009\end{array}\right)$

Up to an unknown scaling factor $\mu<0$, there is a unique solution for $R$, for which $\pi$ is the steady-state of $Q$ on the basis of $K$ and (13). This solution $r \in I R^{6}$ is given by the linear equation

$r^{T} D(K-i d)=0$ 
where $K$ is given by the Arrhenius approximation, $D$ is the diagonal matrix $D=\operatorname{diag}(\pi)$ of the given steadystate solution, and $r$ is the unknown vector of rate factors, with $R=\operatorname{diag}(r)$, resulting in

$$
Q^{\text {theo }}=\mu \cdot\left(\begin{array}{cccccc}
-0.0192 & 0.0002 & 0.0191 & 0 & 0 & 0 \\
0.0022 & -0.1999 & 0 & 0 & 0.1971 & 0.0006 \\
0.9989 & 0 & -1 & 0 & 0.0011 & 0 \\
0 & 0 & 0 & -0.192 & 0.0002 & 0.0191 \\
0 & 0.1971 & 0.0006 & 0.0022 & -0.1999 & 0 \\
0 & 0.0011 & 0 & 0.9989 & 0 & -1
\end{array}\right) \text {. }
$$

For more details about the determination of $Q$, see [34]. A simulation of the kinetics (1) using this matrix is shown in Figure 7 , where $\mu=0.001$ has been fixed "by eye". Note that $\mu$ is not available via classical methods, due to the unknown velocity of the cyclic-concerted isomerisation step (Figure 3). The simulation was performed for the initial condition of the kinetic experiment (Figure 5). Although a number of assumptions had been made (classical approach, vacuum, Boltzmann distribution, reweighting to $T=433 \mathrm{~K}$, Arrhenius equation, independence of the cyclic-concerted isomerisation step from the alignment of the rests $\mathrm{R}$ ...), the simulated rates correctly describe the qualitative behavior of the system (Figure 7). 

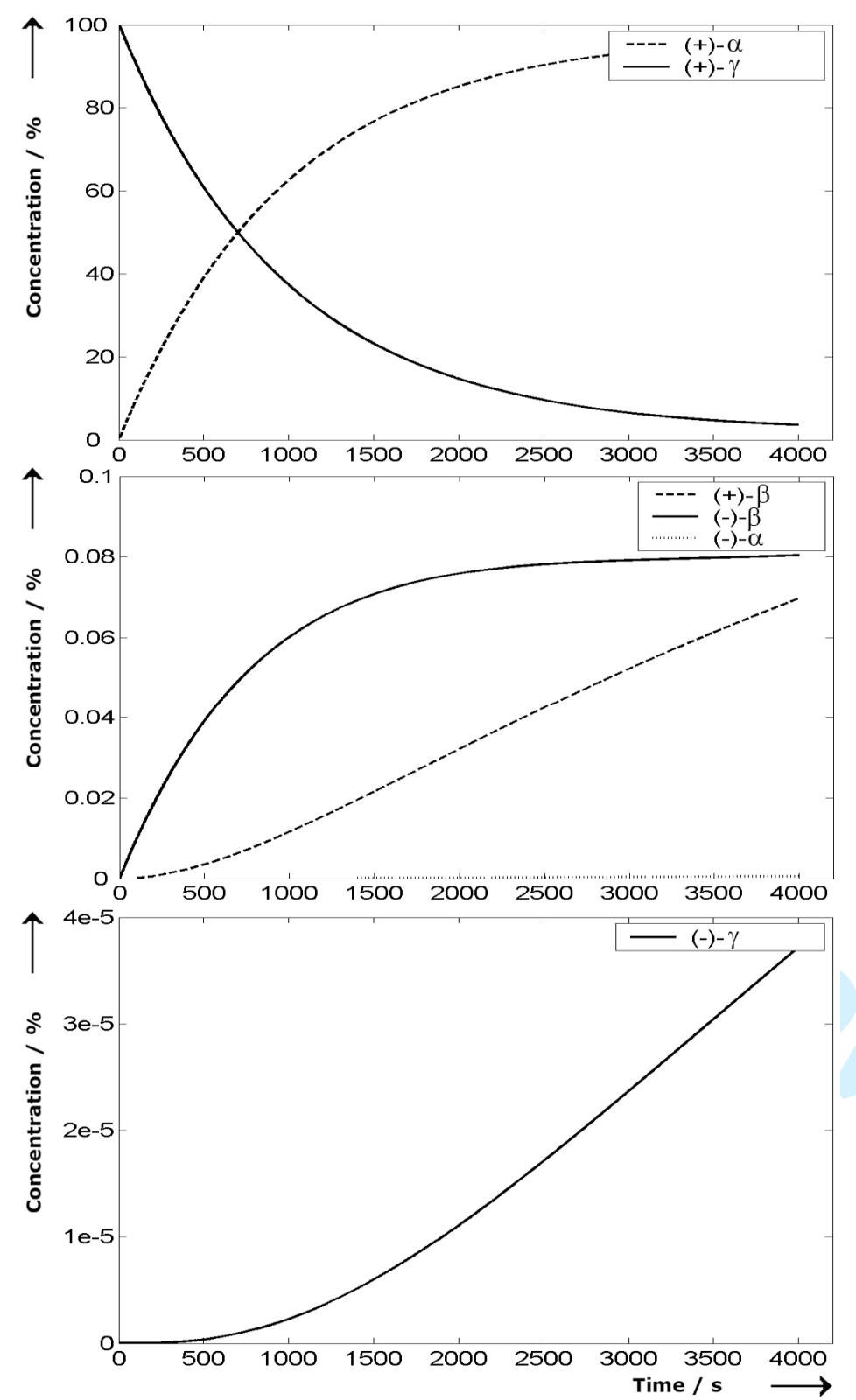

Figure 7. Qualitative prediction of the interconversion based on computed free energies.

The differing increases of both, the (+)- $\beta$-HBCD and the $(-)-\beta$-HBCD concentration, the initial increase of the (+)- $\alpha$-HBCD concentration being far above its equilibrium value, and the low initial concentrations of $(-)-\alpha-$ HBCD and $(-)-\gamma$-HBCD. Thus, we can conclude that the numerical simulations reflect qualitatively the experimentally determined behaviour of the HBCD-system, as shown in Figure 5.

A detailed comparison of $K$ and $Q^{\text {theo }}$ with the experimental results $\left(Q^{\text {exp }}\right.$ in the Experimental Section) provides the following analogies: The dominating reaction is the interconversion from $(+)-\gamma-$ to $(+)-\alpha$-HBCD followed by the racemisation from $(+)-\beta$ - into $(-)-\beta$-HBCD. The other interconversion reactions are much slower. The severest approximation in our classical model may be the vacuum-approximation. Liquid HBCD has a dielectric constant which is different from the vacuum. Also, the interactions between the HBCD molecules in a liquid phase can not be neglected. Briefly, the energy barriers and the energy differences are 
overestimated by the classical model in this section. From a statistical point of view, a simple downscaling of the potential energy function is equivalent to increasing the temperature. And, in fact, for a temperature of $T=$ $670 \mathrm{~K}$, we get the steady-state estimation:

$$
\pi^{T}=\left(\begin{array}{llllll}
0.391 & 0.065 & 0.043 & 0.391 & 0.065 & 0.044
\end{array}\right)
$$

which even quantitatively fits to experimental results [10] (78\% $\alpha$-HBCD, $14 \% \beta$-HBCD and $8 \% \gamma$-HBCD). Furthermore, for this medium temperature, the main interconversion rates $(\alpha \leftrightarrow \gamma$ and $\beta \leftrightarrow \beta)$ with $\mu=0.0015$ also quantitatively fit to the experiment:

$$
Q^{\text {theo }}=0.001 \cdot\left(\begin{array}{cccccc}
-0.1730 & 0.0084 & 0.1646 & 0 & 0 & 0 \\
0.0405 & -0.9764 & 0 & 0 & 0.9152 & 0.0208 \\
1.4843 & 0 & -1.5 & 0 & 0.0157 & 0 \\
0 & 0 & 0 & -0.1730 & 0.0084 & 0.1646 \\
0 & 0.9152 & 0.0208 & 0.0405 & -0.9764 & 0 \\
0 & 0.0157 & 0 & 1.4843 & 0 & -1.5
\end{array}\right) \text {. }
$$

These values correspond to the following rates in $\left(\right.$ mol- $\left.\% \mathrm{~s}^{-1}\right): \mathrm{k}_{\alpha \rightarrow \gamma}=1.65 \cdot 10^{-4}, \mathrm{k}_{\alpha \rightarrow \beta}=8.4 \cdot 10^{-6}, \mathrm{k}_{\beta \rightarrow \alpha}=$ $4.1 \cdot 10^{-5}, \mathrm{k}_{\beta \rightarrow \gamma}=2.1 \cdot 10^{-5}, \mathrm{k}_{\gamma \rightarrow \beta}=1.6 \cdot 10^{-5}, \mathrm{k}_{\gamma \rightarrow \alpha}=1.48 \cdot 10^{-3}, \mathrm{k}_{\beta \rightarrow \beta}=9.2 \cdot 10^{-4}$.

\section{Conclusion and outlook}

The interconversion reactions of HBCD are quantum mechanical processes which, due to the timescale of seconds, cannot be investigated by quantum mechanical molecular simulation. However, since the quantum mechanical mechanism is identical for all interconversion processes of HBCD, it is only important to know which part of the conformational space initializes the process. Then, based on classical simulations, one can compute the portion of the conformational space which can be seen as activated. This portion is a measure for the corresponding transition rate. In case of HBCD, even the classical simulation algorithm has to tackle very different timescales and only high temperature HMC-samplings are able to provide correct thermodynamical ensembles. We have presented a simple reweighting formula to interpolate the important thermodynamical values at lower temperature. With all these simplifications, the HMC-samplings for each of the HBCDstereoisomers required 30 million force field evaluations (CPU-time on our simple PC: 6 hours). After applying a reweighting scheme, the samplings nicely reflect the experimental results. The presented ideas use the fact that the unknown rate of the cyclic-concerted rearrangement is identical for each transition between HBCD stereoisomers. In this case, the time scale factor $\mu$ is the only unknown value in the rate matrix computation. If the interconversion mechanism is different for each of the interconversion processes, the above scheme for the rate matrix computation is also valid. In this case, we have to estimate the activation energy of the corresponding cyclic-concerted rearrangement, e.g., by the means of quantum-based transition state computations. This additional activation energy has to be inserted into the Arrhenius equation (12), too, leading us to a combination of single-point quantum chemistry calculations with results of classical thermodynamics simulations. 


\section{Acknowledgements}

This work was supported in the framework of the BAM-ZIB cooperation on common research and development in the field of scientific computing. Date of agreement: July 10th, 2007.

\section{References}

[1] A. Covaci, A.S.C. Gerecke, R.J. Law, S. Voorspoels, M. Kohler, N.V. Heeb, H. Leslie, C.R. Allchin, J. De Boer. Hexabromocyclododecanes (HBCDs) in the environment and humans: a review. Environ. Sci. Technol., 40, 3680 (2006).

[2] C.A. de Wit. An overview of brominated flame retardants in the environment. Chemosphere, 40, 583 (2002).

[3] C.A. de Wit, M. Alaee, D.C.G. Muir. Levels and trends of brominated flame retardants in the arctic. Chemosphere, 64, 209 (2006).

[4] K. Law, V.P. Palace, T. Halldorson, R. Danell, K. Wautier, B. Evans, M. Alaee, C. Marvin, G.T. Tomy. Dietary accumulation of hexabromocyclododecane diastereoisomers in juvenile rainbow trout (Oncorhynchus mykiss) I: Bioaccumulation parameters and evidence of bioisomerization. Environ. Toxicol. Chem., 25, 1757 (2006).

[5] J.G. Vos, G. Becher, M. van den Berg, M. de Boer, P.E.G. Leonards. Brominated flame retardants and endocrine disruption. Pure Appl. Chem., 75, 2039 (2003).

[6] R. Koeppen, R. Becker, F. Emmerling, C. Jung, I. Nehls. Enantioselective preparative HPLC separation of the HBCD-stereoisomers from the technical product and their absolute structure elucidation using x-ray crystallography. Chirality, 19, 214 (2007).

[7] B.N. Zegers, A.Mets, R. van Bommel, C. Minkenberg, T. Hamers, J.H. Kamstra, G. J. Pierce, J.P. Boon. Levels of Hexabromocyclododecane in Harbor Porpoises and Common Dolphins from Western European Seas, with Evidence for Stereoisomer-Specific Biotransformation by Cytochrome P450. Environ. Sci. Technol., 39, 2095 (2005).

[8] M. Peled, R. Scharia, D. Sondack. Thermal rearrangement of hexabromocyclododecane (HBCD), in Advances in Organobromine Chemistry, J.R. Desmurs, B. Gerard, M.J. Goldstein, eds., Elsevier, Amsterdam, 92 (1995).

[9] K. Janak, A. Covaci, S. Voorspoels, G. Becher. Hexabromocyclododecane in marine species from the western Scheldt estuary: Diastereoisomer- and enantiomerspecific accumulation. Environ. Sci. Technol., 39, 1987 (2005).

[10] R. Köppen, R. Becker, C. Jung, I. Nehls. On the thermally induced isomerisation of hexabromocyclododecane stereoisomers. Chemosphere, 71, 656 (2008).

[11] N.V. Heeb, W.B. Schweizer, P. Mattrel, R. Haag, M. Kohler, P. Schmid, M. Zennegg, M. Wolfensberger, Regio- and stereoselective isomerization of hexabromocyclododecanes (HBCDs): Kinetics and mechanism of $\beta$-HBCD racemization. Chemosphere 71, 1547, (2008).

[12] C.A. Grob, S. Winstein. Mechanismus der mutarotation von 5,6-dibromcholestan. Helv. Chim. Acta., 35, 780 (1952).

[13] J.F. King, R.G. Pews. Reaction mechanism studies: 3. solvent effects and nature of transition state in diaxial $\rightarrow$ diequatorial rearrangement. Can. J. Chem., 43, 847 (1965).

[14] P.L. Barili, G. Bellucci, G. Berti, A. Marioni, A. Marsili, I. Morelli. Thermal equilibrium of substituted trans1,2-dibromocyclohexanes. J. Chem. Soc. Perk. Trans. 2(1), 58 (1972).

[15] G. Bellucci, A. Marsili, E. Mastrorilli, I. Morelli, V. Scartoni. Kinetics of thermal racemization of some 1,2dihalides. J. Chem. Soc. Perkin Trans., 2(2), 201 (1974).

[16] T.A. Halgren. The representation of Van der Waals (VdW) interactions in molecular mechanics force fields: potential form, combination rules, and vdW parameters. J. Am. Chem. Soc., 114, 7827 (1992).

[17] T.A. Halgren. Merck molecular force field. J. Comp. Chem., 17, 490 (1996).

[18] S. Duane, A.D. Kennedy, B.J. Pendleton, D. Roweth. Hybrid Monte Carlo. Phys. Lett. B, 195, 216 (1987).

[19] C. Schütte, A. Fischer, W. Huisinga, P. Deuflhard. A direct approach to conformational dynamics based on hybrid Monte Carlo. J. Comput. Phys., Special Issue on Computational Biophysics, 151, 146 (1999). 
[20] D. Frenkel, B. Smit, Understanding Molecular Simulation - From Algorithms to Applications, in Computational Science Series, Vol. 1, Academic Press (2002).

[21] A. Fischer. An Uncoupling-Coupling Method for Markov Chain Monte Carlo Simulations with an Application to Biomolecules. Doctoral thesis, Freie Universität Berlin (DE) (2003).

[22] A. Fischer, C. Schütte, P. Deuflhard, F. Cordes. Hierarchical uncoupling-coupling of metastable conformations, in Computational Methods for Macromolecules: Challenges and Applications, Proc. of the 3rd Intern. Workshop on Algorithms for Macromolecular Modelling, T. Schlick, H.H. Gan, eds., Springe, Berlin, Heidelberg, New York, 235 (2002).

[23] A. Gelman, D. Rubin. Inference from Iterative Simulation using Multiple Sequences. Statist. Sci., 7, 457 (1992).

[24] A. Gelman, D. Rubin. Markov chain Monte Carlo Methods in Biostatistics. Meth. Med. Res., 5, 339 (1996).

[25] Y. Sugita, Y. Okamoto. Replica-exchange molecular dynamics method for protein folding. Chem. Phys. Letters, 314, 141 (1999).

[26] Ch. Chipot, A. Pohorille. Free Energy Calculations, eds. in Springer Series in Chemical Physics, Vol. 86, Springer, Berlin, Heidelberg (2007).

[27] M.R. Hestens, E. Stiefel. Methods of Conjugate gradients for Solving Linear Systems. J. Res. Nat. Bur. Stand., 49, 409 (1952).

[28] N.V. Heeb, W.B. Schweizer, M. Kohler, A.C. Gerecke. Structure elucidation of hexabromocyclododecanes - a class of compounds with a complex stereochemistry. Chemosphere, 61, 65 (2005).

[29] N.V. Heeb, W.B. Schweizer, P. Mattrel, R. Haag, M. Kohler. Crystal structure analysis of enantiomerically pure (+) and (-)-hexabromocyclododecanes. Chemosphere, 66, 1590 (2007).

[30] N.V. Heeb, W.B. Schweizer, P. Mattrel, R. Haag, A.C. Gerecke, M. Kohler, P. Schmid, M. Zennegg, M. Wolfensberger. Solid-state conformations and absolute configurations of $(+)$ and $(-) \alpha-, \beta-$, and $\gamma-$ hexabromocyclododecanes (HBCDs). Chemosphere, 68, 940 (2007).

[31] W. Göpel, H.-D. Wiemdörfer. Statistische Thermodynamik. Spectrum, Akademischer Verlag (2000).

[32] S. Kube, M. Weber. Conformation Kinetics as a Reduced Model for Transition Pathways, ZIB report 05-43, Zuse Institute Berlin (2005).

[33] M. Kijima. Markov Processes for Stochastic Modeling, in Stochastic Modelling Series, Chapman and Hall (1997).

[34] M. Weber. Conformation-Based Transition State Theory. ZIB report 07-18, Zuse Institute Berlin (2007).

[35] M. Weber. Meshless Methods in Conformation Dynamics, Doctoral thesis, Freie Universität Berlin, 2006.

[36] M. Weber, S. Kube, L. Walter, P. Deuflhard. Stable computation of probability densities for metastable dynamical systems. SIAM J. Multisc. Mod. Sim., 6, 396 (2007).

\section{Appendix}

\section{Temperature dependence of the mean potential energy}

In order to justify the linear model for the mean potential energy in (8), it will be shown that this behaviour is valid for quadratic potentials $V_{p o t}: I R^{d} \rightarrow I R$. This also justifies the application of a linear model for multivariate Boltzmann distributions which are locally Gaussian (approximately locally quadratic potential energy functions). Without loss of generality (and via principle axis transformation), the $d$-dimensional quadratic potential energy function can be written in separable form

$$
V_{p o t}(q)=\sum_{i=1}^{d}\left(a_{i} q_{i}^{2}+b_{i} q_{i}+c_{i}\right):=\sum_{i=1}^{d} V_{i}\left(q_{i}\right)
$$


with corresponding constants $a_{\mathrm{i}}, b_{\mathrm{i}}, c_{\mathrm{i}} \in I R$ and $a_{\mathrm{i}}>0$. A short calculation on the basis of (6) and (15) yields

$$
\left\langle V_{p o t}\right\rangle=\sum_{i=1}^{d} \int_{-\infty}^{\infty} V_{i}\left(q_{i}\right) \frac{\exp \left(-\beta V_{i}\left(q_{i}\right)\right)}{\exp \left(-\beta V_{i}\left(q_{i}\right)\right) d q_{i}} d q_{i} .
$$

Equation (16) shows that the linear dependency of the mean potential energy can be justified by solving the integral in (6) for the 1 -dimensional case. The result is

$$
\left\langle V_{p o t}\right\rangle=\sum_{i=1}^{d} \frac{1}{2 \beta}-\frac{b_{i}^{2}}{4 a_{i}}+c_{i}
$$

which is exactly the claimed $\beta^{-1}$-behavior of $\left\langle V_{\text {pot }}\right\rangle$. With $b_{\mathrm{i}}=0$ and $c_{\mathrm{i}}=0$, equation (17) is the well-known $0.5 \mathrm{kT}$ contribution per degree of freedom to the inner energy of an ideal gas. For a numerical justification of the linear temperature dependence, we performed two HMC samplings of $(+)-\alpha$-HBCD at $T=433 \mathrm{~K}$. One sampling started in the low energy region of the optimal conformation of $(+)-\alpha$-HBCD and another sampling in a high-energy region. After five runs with 10,000 steps the samplings were halted. The mean potential energy values were $420 \mathrm{~kJ} \mathrm{~mol}^{-1}$ and $455 \mathrm{~kJ} \mathrm{~mol}^{-}$ ${ }^{1}$, respectively. They are lower and upper bounds for the true value. From the mean potential energy $\left\langle V_{\text {pot }}\right\rangle=921 \mathrm{~kJ}$ $\mathrm{mol}^{-1}$ at high temperature and an optimal value $V_{\text {pot }}{ }^{0}=239 \mathrm{~kJ} \mathrm{~mol}^{-1}$ of the potential energy, the linear model (8) predicts a mean energy of $435 \mathrm{~kJ} \mathrm{~mol}^{-1}$ at a temperature of $433 \mathrm{~K}$ in accordance with the simulated bounds. For a better verification of the reweighting formula, we performed an extensive simulation with the in-house software ZIBgridfree [35] and a pointwise density estimation strategy [36]. The simulated mean potential energy value of $434 \mathrm{~kJ}^{\mathrm{mol}}{ }^{-1}$ is very close to the calculated value of $435 \mathrm{~kJ} \mathrm{~mol}^{-1}$ of the reweighting formula. For all these reasons, we think that the reweighting formula is applicable. 